\title{
EVALUATION OF SOIL SALINITY IN BATIAGHATA UPAZILA
}

\author{
Nur A Faisal \\ Soil, Water and Environment \\ Khulna University, Khulna
}

\author{
Baig Abdullah Al Shoumik \\ Soil, Water and Environment \\ Khulna University, Khulna
}

\author{
Md. Sanaul Islam \\ Soil, Water and Environment \\ Khulna University, Khulna
}

\begin{abstract}
In Bangladesh, almost 53\% of the coastal areas are severely affected by salinity which is the major cause for unfavorable environment and hydrological conditions. This unfavorable condition in turn restricts the usual and normal crop production. Batiaghata, an upazila of Khulna in Bangladesh is one of the coastal areas which are severely affected by salinity. This research was conducted to evaluate the soil salinity level (EC value) in 2018 and to compare that collected data with the data obtained by SRDI in 2008 from the similar sites. It was found that the sampling sites that had been urbanized in 2018 showed lesser EC value than 2008 while sampling sites where rice cultivation had been practiced since 2008, showed an increment in $\mathrm{EC}$ value.
\end{abstract}

Keywords - Agricultural Land; Batiaghata Upazila; EC Value; Soil Salinity

\section{INTRODUCTION}

As a deltaic country, $80 \%$ area of Bangladesh consists of alluvial sediments that are deposited by the rivers Ganges, Brahmaputra, Tista, Jamuna, Meghna and their tributaries [1]. Around $53 \%$ of the coastal areas are affected by salinity which causes unfavorable environment and hydrological situation that restrict the normal crop production throughout the year [2]. The severity of salinity problem in Bangladesh increases with the desiccation of the soil and there are several factors like tidal flooding during wet season (June - October), direct inundation by saline water and upward movement of saline ground water are responsible for salinity development in coastal regions [3].

Karim et al., [2] classified soil salinity class based on the range of electrical conductivity (EC) of soils. He classified EC value of 0-2 dS/m, 2-4 dS/m, 4-8 dS/m, 8-16 dS/m and >16 $\mathrm{dS} / \mathrm{m}$ as Non saline, Slightly saline, Moderately saline, Saline, Highly saline respectively.

Batiaghata upazila, located at the south-west coastal region of Khulna district is a heavily saline affected area. Shammi et al., [4] found that the EC level of all the water samples collected from groundwater and surface water were slightly higher than the acceptable limit in the monsoon. Corwin et al., [5] revealed that the irrigation water quality in the south-west coastal region of Bangladesh is highly affected by salinity problem due to the lack of adequate leaching and drainage and the presence of high groundwater table.

Since 2008, changes in salinity level have been taking place in Batiaghata upazila and therefore people in remote areas are now migrating towards urban areas. Soil Resource Development Institute (SRDI) investigated the salinity level in Khulna in 2008. However, Batiaghata is now lot more developed and urbanized, which may change the salinity level in this upazila.

Therefore, this study was conducted to evaluate the salinity level in 2018 to observe the changes in salinity level from 2008 and to upgrade soil salinity map in Batiaghata upazila.

\section{METHODS AND MATERIALS}

The study area Batiagahta upazila lies between $22^{\circ} 39^{\prime}$ to $22^{\circ} 54^{\prime}$ north latitude and $89^{\circ} 15^{\prime}$ to $89^{\circ} 32^{\prime}$ east longitude. Due to the presence of different ranges of saline soil and the inclusion of the saline water, this upazila was selected as the experimental site.

The upazila lies in the physiographic unit of high ganges alluvium floodplain and ganges tidal floodplain. Major land type in this upazila is medium highland to medium lowland and the textural class is mainly clay loam. The upazila is consisted of 7 unions among which 4 unions are major and sampling was done from these 4 unions, and they are Jalma Union, Gangarampur Union, Batiaghata Union and Surkhali Union. On the other hand, the remaining three Amirpur Union, Baliadanga Union, and Bhanderkote Union were remote areas and due to inaccessibility, sampling was not possible from there.

\section{RESULT AND DISCUSSION}

The electrical conductivity of four unions named Batiaghata, Jalma, Gangarampur and Surkhali and the comparison between the obtained EC in 2018 and the EC obtained by SRDI in 2008 is represented in Fig. 1 to Fig. 4.

Fig. 1 demonstrates the comparison of EC value in 2008 and 2018, and among 27 sampling sites, the EC value has decreased in 11 sites in 2018 while the rest 16 sites show the opposite. The decreased 11 sites named B1, B2, B4, B14, B15, B16, B17, B18, B23, B24 and B26 were the urbanized area [6] while the rest 16 points are from cropping land with the major cropping pattern of "Rice-Fallow-Fallow". 
Fig. 2 shows that among 31 sampling points; only 2 sites J26 and $\mathbf{J} 27$ show increased EC level while the remaining 29 points has decreased EC level in 2018. Based on the map, provided by the SRDI [6], all the areas having decreased EC in 2018 are urbanized while the remaining 2 areas were cropping field where the major cropping patter was "Rice-Lentil-Rice".

Fig. 3 demonstrates that among 15 sampling areas, 8 areas has increased EC value while the rest 7 has decreased EC value in 2018. In this union, the urbanized areas are G2, G3, G5, G6, G7, G8 and G3 [6] while G1, G4, G9, G10, G1, G12, G14 and G15 are the cropping land having the main cropping pattern of
"Rice-Fallow-Rice" which has resulted in an increased EC level.

Fig.4 illustrates that all of the sampling sites show higher EC than 2008 and the reason behind this increment can be the practice of cropping pattern which is "Rice-Fallow-Rice". Surkhali is a rural area and is not so urbanized. The collected 11 samples were from the same cropping field and same points where SRDI in 2008 collected as well. The obtained results from the analysis showed that the EC level has increased for every sampling point than 2008 .

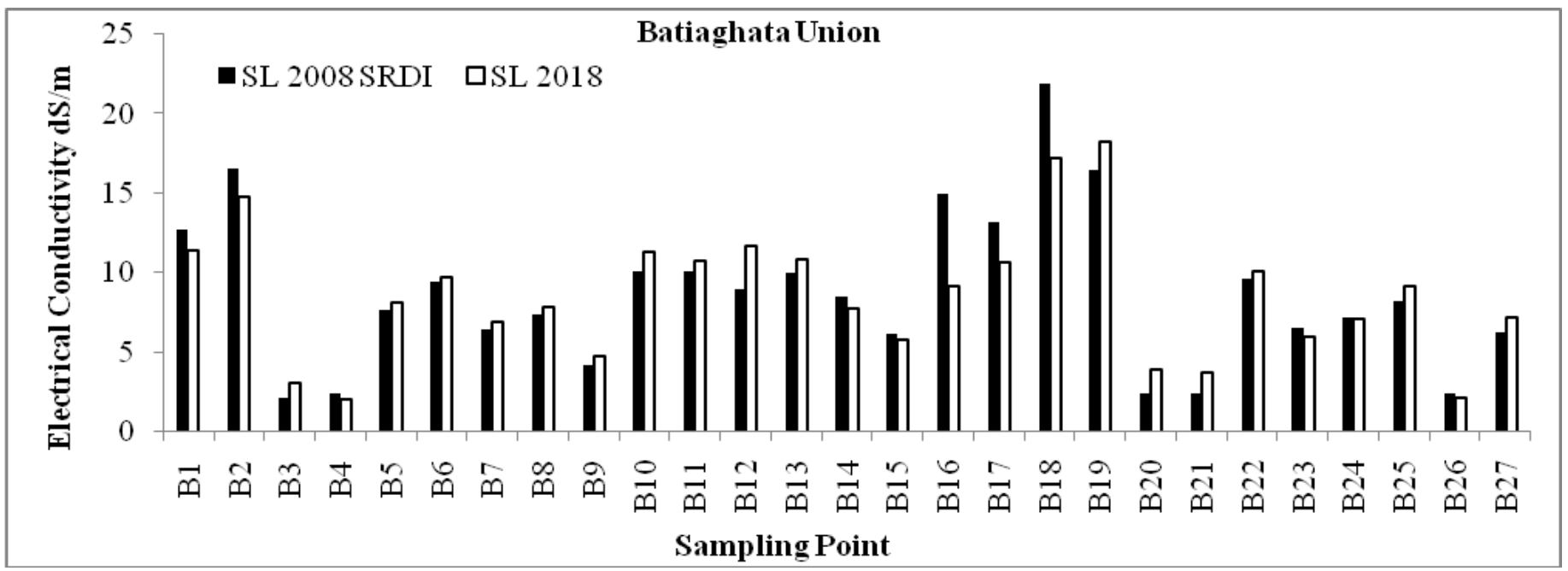

Fig. 1. EC Level of Batiaghata Union of same point in 2008 and 2018.

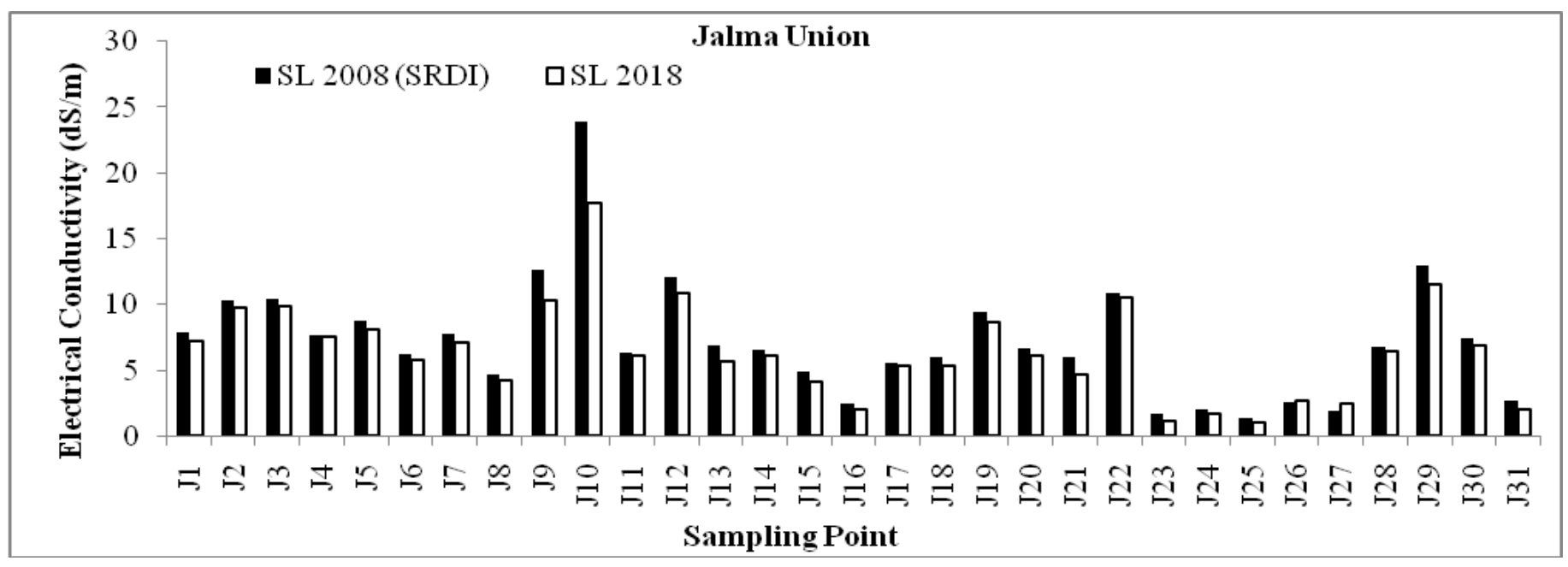

Fig. 2. EC Level of Jalma Union of same point in 2008 and 2018. 


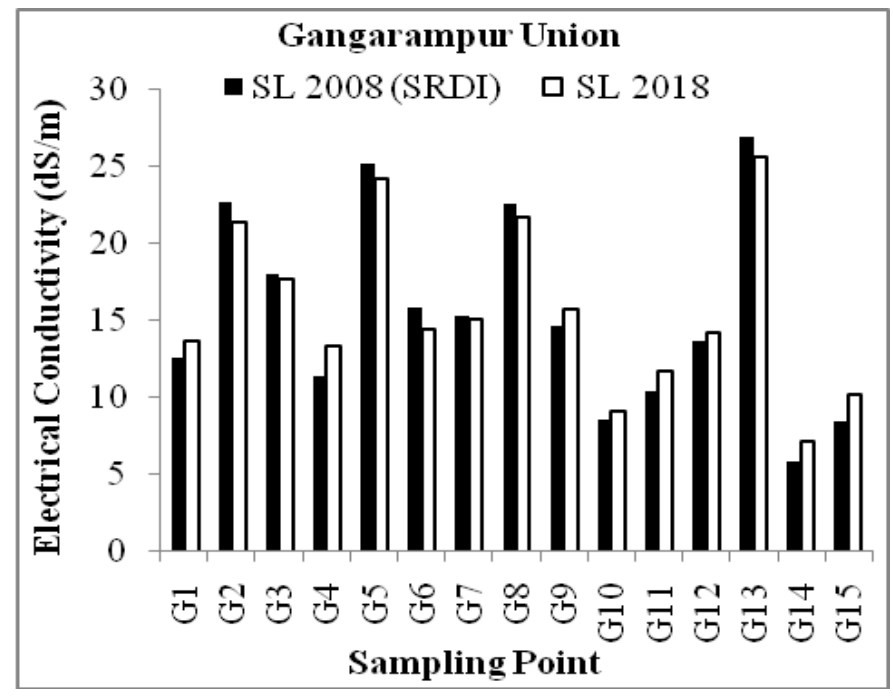

Fig. 3. EC Level of Gangarampur Union of same point in 2008 and 2018.

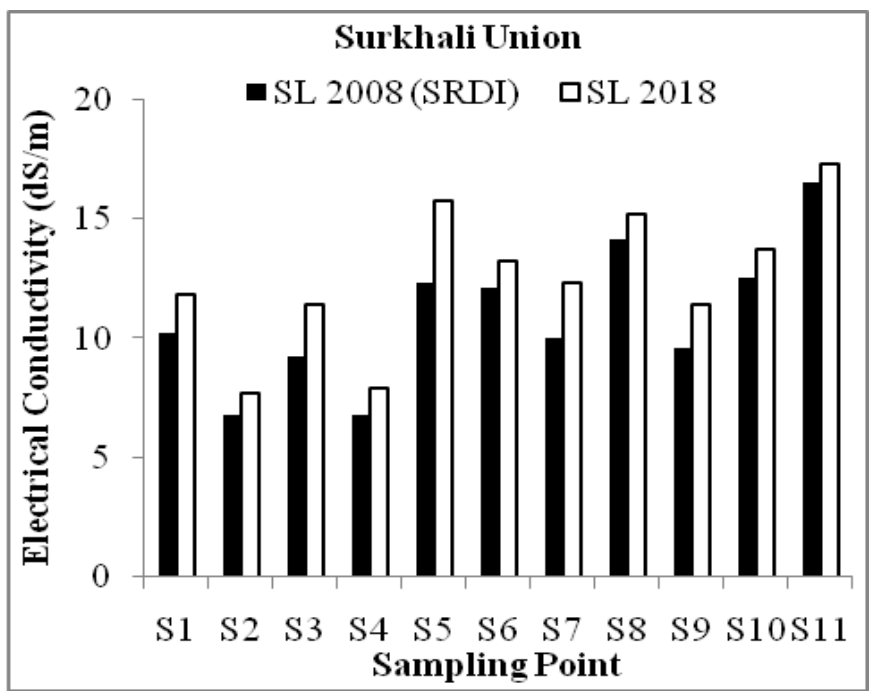

Fig. 4. EC Level of Surkhali Union of same point in 2008 and 2018.

It has been observed that the sampling points which were under rice cultivation have showed the increment in EC value. Besides, the local farmers used groundwater for their irrigation purpose that contained high level of salinity as explained by Corwin et al., [5]. This utilization of saline water for irrigation purpose could be the reason of this increment in salinity level. Furthermore, the sampling was done during the pre-monsoon period, so, there was a chance for the presence of higher EC value in the irrigation water which might increase the level of salinity in the field.

On the other hand, the sampling sites that are now under concrete and are urbanized have showed reduction in the EC value. The reason behind the reduction can be the 'Batiaghata Protection Embankment' or polderization which was introduced by Bangladesh government to protect the coastal areas from salinity intrusion. The polderization has prevented the flow of highly saline river water to enter into the polders and in addition, these polders only receive the rain water which is not saline. Probably, this is the reason why soil salinity in these urbanized areas has decreased than 2008.

\section{CONCLUSION}

Under the scope of this study, a total of 84 sampling points under 4 unions of Batiaghata upazila named Jalma (31), Batiaghata (27), Gangarampus (15) and Surkhali (11) were covered. Among these unions, Surkhali showed a higher increment and change in salinity level. However, it has been observed that sampling sites inside the embankment show lesser EC value than before.

\section{REFERENCE}

[1] Haque, S.A. 2006. Salinity problems and crop production in coastal regions of Bangladesh. Pakistan Journal of Botany, 38(5): 1359-1365.

[2] Karim, Z.; Hussain, S.G. and Ahmed, M. 1990. Salinity problems and crop intensification in the coastal regions of Bangladesh. Soils publication No. 33. BARC. pp. 17.

[3] Karim, Z.; Saheed, S.M.; Salauddin, A.B.M.; Alam, M.K. and Huq, A. 1982. Coastal saline soils and their management in Bangladesh. Soils publication No. 8. BARC. pp. 33.

[4] Shammi, M.; Karmakar, B.; Rahman, M.M.; Islam, M.S.; Rahman, R. and Uddin, M.K. 2016. Assessment of Salinity Hazard of Irrigation Water Quality in Monsoon Season of Batiaghata Upazila, Khulna District, Bangladesh and adaptation strategies. Pollution, 2(2): 183-197.

[5] Corwin, D.L.; Rhoades, J.D. and Šimůnek, J. 2007. Leaching requirement for soil salinity control: Steadystate versus transient models. Agricultural Water Management, 90(3): 165-180. DOI: https://doi.org/10.1061/9780784411698.ch26.

[6] Soil Resource Development Institute (SRDI), 2008. Land and Soil Resource Utilization Guide (Upazila Nirdeshika, in Bengali): 91 vol of 18 districts, Khulna BarisalChittagong Divisions. 